This document is published in:

Molina, J. M. et al. (eds.) (2011) User-Centric Technologies and Applications: Proceedings of the CONTEXTS 2011 Workshop. (Advances in Intelligent and Soft Computing, 94). Springer, 59-68. DOI: http://dx.doi.org/10.1007/978-3-642-19908-0_7

(C) 2011 Springer-Verlag Berlin Heidelberg 


\title{
Opportunistic Multi-sensor Fusion for Robust Navigation in Smart Environments
}

\author{
Enrique Martí, Jesús García, and José M. Molina \\ Group of Applied Artificial Intelligence, Universidad Carlos III de Madrid, \\ Av. de la Universidad Carlos III, 22, 28270 Colmenarejo, Madrid, Spain \\ e-mail: emarti@inf.uc3m.es, jgherrer@inf.uc3m.es, \\ molina@ia.uc3m.es
}

\begin{abstract}
This paper presents the design of a navigation system for multiple autonomous robotic platforms. It performs multisensor fusion using a Monte Carlo Bayesian filter, and has been designed to maximize information acquisition. Apart from sensors equipped in the mobile platform, the system can dynamically integrate observations from friendly external sensing entities, increasing robustness and making it suitable for both indoor and outdoor operation. A multi-agent layer manages the information acquisition process, making it transparent for the core filtering solution. As a proof of concept, some preliminary results are presented over a real platform using the part of the system specialized in outdoor navigation.
\end{abstract}

Keywords: multi agent, sensor fusion, positioning, indoor/outdoor navigation, particle filter.

\section{Introduction}

In the last years we have seen several implementations of autonomous robotic platforms doing simple works or assisting humans in theirs. These realizations have taken place in environments as disparate as hospitals [1] or factories[2], and, in spite of their relative simplicity, they can be seen as an advance of a future in which robotic workers will be massively used in more complex tasks. In view of such potential scenario, both self-location and navigation are problems of the uttermost importance for achieving continued, fail-proof operation.

This work aims to introduce a simple but robust architecture for combined indoor/outdoor navigation through sensor fusion technology, where the information provided by on-board sensors is aligned with heterogeneous external references [3] coming from different sources.

The core of the navigation solution is implemented as a Sampling Importance Resampling (SIR) Particle Filter (PF) [4] with loose coupling integration of 
received information. Apart from being capable of dealing with the non-linear relation between internal sensors and absolute external references, this Bayesian inference tool requires minimal efforts for integrating new types of sensor measures -to the point of not needing previous knowledge about the sensor: just the type of data it is providing and a description of the associated uncertainty.

Our proposal achieves accuracy and reliability through redundancy, considering the set of sensors to be fused as a changing entity. Apart from the typical internal devices -IMU, laser, GPS, odometry-, the robotic platform is able to obtain additional information from external entities, such as Ultra Wide Band sensor networks or external video-based trackers.

Works on navigation through multisensor fusion usually define architectures and algorithms specifically tailored for a selected set of sensors, as in the cases of tight coupling or feature based navigation[5].Those specialized approaches can take advantage of the existing synergies between different sensing technologies, so that the final result is more stable, accurate or computationally affordable. Nonetheless, it is more sensitive against changes in the set of sensors: integrating new technologies can be a difficult task, and if an existing device suffers a temporal outage, the system could even be unable to continue its normal function.

Regarding the decision of integrating external sensors, some researchers point to the convenience of pure standalone robots arguing that ad-hoc sensor networks are expensive, could be unavailable, and same or better results can be obtained using internal devices [6]. Our proposal is, however, based in the fact that communication and sensor networks are more common every day. Moreover, multiple robotic platforms coexisting in an environment can be simultaneously benefited from them. This means that the proportional cost of such installation has to be divided and the amount of internal sensors on each mobile unit can be cut back with the subsequent expenses reduction.

The central part of this document contains a detailed description of the proposed system, while the last sections report the results obtained with a first partial implementation capable of outdoor navigation using an Inertial Measure Unit (IMU) and a GPS device. A section with some conclusions and projected future work closes the document.

\section{Description of the Proposed System}

The system can be split into two parts: the sensor fusion process inside a single robotic mobile platform, and the cooperative network formed by several of these devices together with an intelligent environment. Let us begin with the internals of autonomous mobile platforms.

\subsection{Fusion Architecture for a Single Platform}

From the architectural point of view, the navigation system of each robot is organized in layers. Each tier plays a different role in the process of acquiring 
information, and transforming it into something useful for the sink of final data: the filtering algorithm. Figure 1. contains a schematic view of the system.

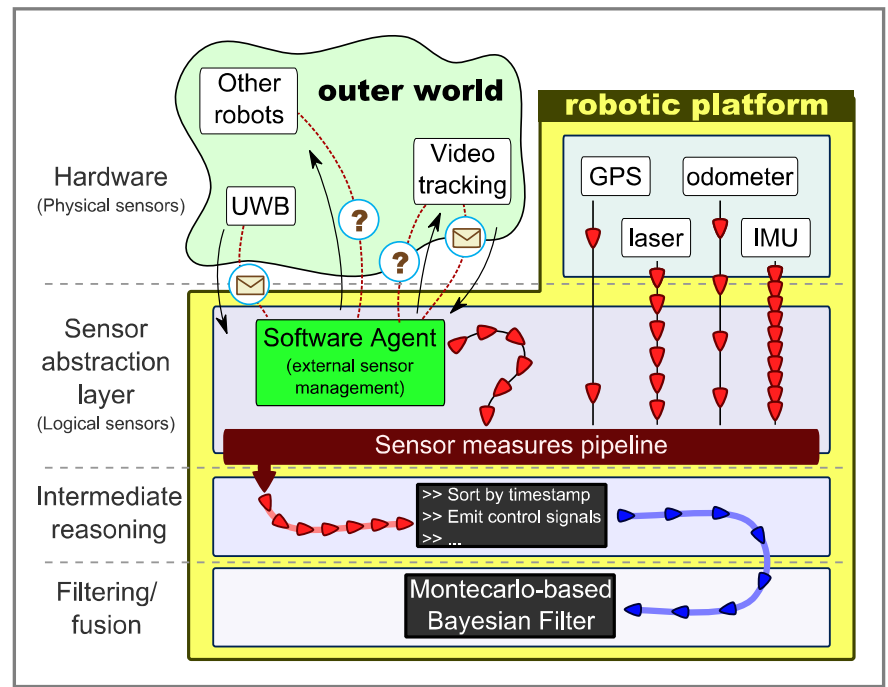

Fig. 1 Architecture of the proposed navigation system for an individual mobile platform

Our solution follows the design principle stated in the introduction of being robust against sensor outages. The selected architecture includes mechanisms for this purpose from the very beginning of the fusion process -data acquisition-to the filtering solution.

Right at the top of the architecture is the Sensor abstraction layer. This level manages communication with both internal (on-board) and external real sensing devices, providing lower levels with a view of logical data sources with unified access interface. Thus, this abstraction level supports online sensor addition and removal, while isolating inferior processes from nasty details and the consequences associated to such changes.

The Intermediate reasoning layer receives bare measures from sensor abstraction level and adapts it to the filter various necessities. Our first implementation is restricted to basic operations required by lower layers, assorting sensor readings according to the time when they were generated. However, it is intended to support advanced processes of high-level reasoning, as the application of fuzzy reasoning to detect sensor malfunction.

In the bottom layer, the Filtering solution has been chosen to have a reactive working profile: incoming sensor data is integrated as it is fed by the superior layer. It does not impose the presence of specific sensors nor a predefined schedule/order for data arrival.

Following subsections cover the multi-agent system proposed for exchanging sensory information, and the selected filtering solution. Sensor abstraction and intermediate reasoning layers do not have dedicated sections because of their simplicity at current development stage. 


\subsection{Multi-agent System for Sensory Information Exchange}

Being the target of our system combined indoor/outdoor navigation, the mobile platforms implementing it must be autonomous and independent: the presence of external entities is not guaranteed, so they can have to operate in standalone mode.

Central data fusion in the own platform is a simple and effective solution. Nonetheless, in the event of finding other entities capable of providing useful information, we are interested in enabling a collaborative behavior to extend navigation capabilities.

Putting these ideas together brings a simple collaboration scenario, where individuals (each Agent) have compatible goals (self-location, navigation) but lack the ability to accomplish them (information about their state). Information is interpreted here as an ability to reach a goal rather than as a resource, because it can be used simultaneously by several agents and do not lose its value when used.

Figure 2. outlines the proposed system as a heterarchical scheme where agents can be information producers, consumers or both. This architecture has been preferred over any type of hierarchy because it gives the system the desired flexibility and robustness.

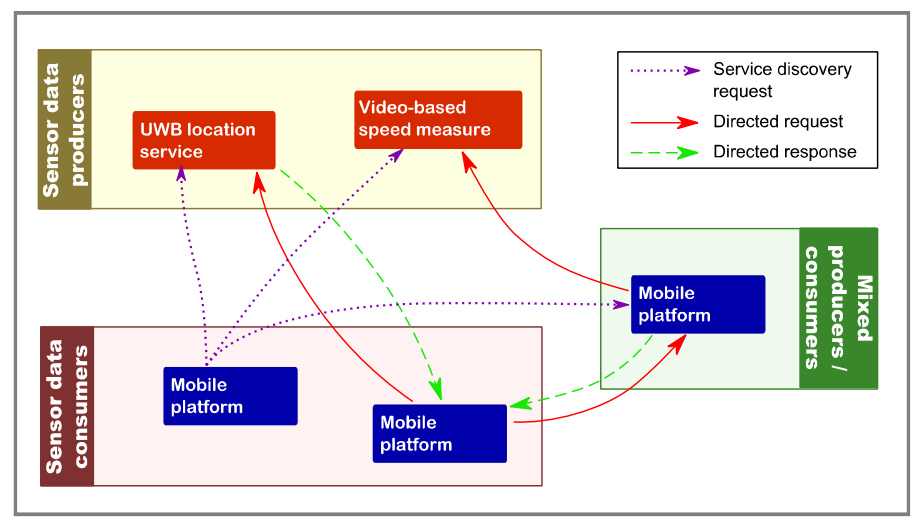

Fig. 2 Schematic example of Multi Agent system for enabling external sensor fusion

Consumers are in charge of asking for new services and requesting sensor measures, to which producer agents can respond or not. In spite of the relative inefficiency of the approach, it offers many advantages in this dynamic scenario: in first place it does not require the existence (and persistent accessibility) of infrastructures as service directories or mediation agents. The second main advantage is that each agent concentrates all the information about its own location/state, so it is the best suited entity for deciding which type information it needs and when to ask it.

Agents will use a simple ontology based in GONZ[7] for information exchange. This ontology has been modified and extended so that it can be used with arbitrary 


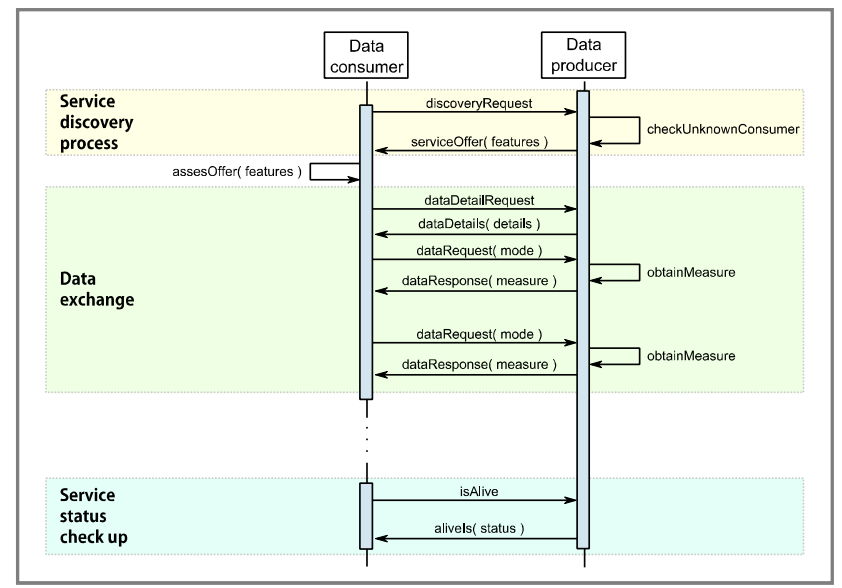

Fig. 3 Interaction diagram showing the caracteristical life cycle of a relation betweena consumer agent (mobile platform) and a producer (external sensor)

types of sensors -not restricted to location services-and includes the additional information required by the fusion algorithm employed in the mobile platforms.

Four pairs of messages are defined for the three types of interaction between agents: service discovery, data exchange and service status check. Almost all communication processes follow a client-server model.

Each action involves the transmission of two messages, the first one making a request (information consumers) and the other responding the petition (information producers). Figure 3. contains all the type of messages available, that we proceed to enumerate and describe. By default (not in the tables) all messages include the unique ID of the sender, the unique ID of the recipient if it is a directed message, and a timestamp.

\begin{tabular}{|cccc|}
\hline discoveryRequest & Field & Description & Sample values \\
\hline (type: broadcast) & & & \\
\hline
\end{tabular}

\begin{tabular}{|clll|}
\hline serviceOffer & Field & Description & Sample values \\
\hline (type: directed $)$ & measureType & Measureable physical magnitudes & PositionXYZ, \\
& & & SpeedXY \\
\hline
\end{tabular}

\begin{tabular}{|c|c|c|c|}
\hline dataDetailRequest & Field & Description & Sample values \\
\hline (type: directed) & & & \\
\hline
\end{tabular}

\begin{tabular}{|llll|}
\hline dataDetails & Field & Description & Sample values \\
\hline $\begin{array}{c}\text { (type: } \\
\text { directed/broadcast) }\end{array}$ & error & Description of error distribution & \\
\hline & samplingFreq & $\begin{array}{l}\text { Maximum frequency with which } 0.01,5 \\
\text { the external sensor can return } \\
\text { measures, in Hz }\end{array}$ \\
\hline & & & \\
\hline
\end{tabular}




\begin{tabular}{|clll|}
\hline dataRequest & Field & Description & Sample values \\
\hline (type: directed) & mode & $\begin{array}{l}\text { How the consumer wants to receive } \\
\text { measures: only under request, or } \\
\text { periodically }\end{array}$ \\
\hline & freq & $\begin{array}{l}\text { Desiredupdate frequency for stream } \\
\text { of measures. Ignored if "request" } \\
\text { was specified in previous field }\end{array}$ \\
\hline
\end{tabular}

\begin{tabular}{|clll|}
\hline dataResponse & Field & Description & Sample values \\
\hline (type: directed) & value & $\begin{array}{l}\text { Array of values according to the }[0.5,3,-1.2] \\
\text { field measureType in the } \\
\end{array}$ & serviceOffer message \\
\hline
\end{tabular}

\begin{tabular}{|cccc|}
\hline isAlive & Field & Description & Sample values \\
\hline (type: directed $)$ & & & \\
\hline
\end{tabular}

\begin{tabular}{|clll|}
\hline aliveIs & Field & Description & Sample values \\
\hline (type: directed $)$ & status & $\begin{array}{l}\text { Availability of the external sensor } \\
\text { to attend requests at this moment }\end{array}$ & $\{$ ready, busy $\}$ \\
\hline
\end{tabular}

The communication scheme has a very simple design, avoiding intricate interaction patterns. Nonetheless, there are two important remarks to be made:

- dataDetails message is the only one that can be sent by information producers without direct request. Changes in the maximum update frequency or in the measure uncertainty can trigger its broadcasting

- The error distribution description in dataDetails message follows its own syntax. Its contents can vary from analytical (multivariate Gaussian distribution: mean and covariance matrix) to sample-based descriptions as that used in [8].

\subsection{Monte Carlo Based Bayesian Filter}

For tracking a mobile platform and integrating all the available information about it, we need a filtering algorithm capable of dealing with non linear models prediction and errors-. The algorithm must also be flexible enough to overcome the difficulty of unstructured data presentation.

The most common filtering algorithms use Bayesian inference to estimate the state of a partially observed system (in our case, position and dynamics of a mobile target). The uncertainty about true state makes necessary to store the belief as a probability distribution, so that at each time the filter can estimate which is the most probable state according to the available information. This probability distribution changes with time. It can be adapted using a prediction model that 
describes system dynamics, and incorporating some occasional measurements providing information about the real state.

Some techniques, as the Kalman Filter (KF) or the Extended Kalman Filter (EKF) [9] assume that all uncertainties have Gaussian distribution, and store the state probability distribution as another Gaussian. Thanks to that simplification, they obtain a matrix-based analytical formulation of the filtering process that can be calculated fast (and is optimal if the assumptions are true).

Nonetheless, if system dynamics obey a highly non-linear model or uncertainties are far from being Gaussian, then these techniques deliver poor performance. A PF[4][10] is a Monte Carlo algorithm capable of dealing with such non-linear non-gaussian scenarios.

Some techniques as the Rao-Blackwellized [11] or the Unscented Particle Filter [12] are known to be more effective than plain PF, but their formulations have subtle details that make more difficult the dynamic integration of heterogeneous sensors. Instead, enabling a Particle Filter for working with a new sensor is as simple as specifying: (a) the sensor error model, (b) function relating state vector with sensor measures (only for sensors providing direct evidences of state), and (c) integration with the prediction model (only if the sensor provides control inputs).

The critical part of our system lays in the procedure for integrating external sensors, about which the mobile platform does not have prior information. Nonetheless, PF can obtain the required data elements as follows: (a) is provided by the own sensor through dataDetails message; (b) is specified by measureType field in serviceOffer message. (c) is not contemplated in our scheme, meaning that external sensors are restricted to provide absolute references.

\section{Outdoor Navigation Experiments}

This last section contains some results implemented over a real platform, intended to assess the suitability of the proposed system under different conditions.

The experiments of this work are based in a GUARDIAN rover from Robotnik corporation [13]. It features a wide range of sensors, including but not limited to odometry, laser ranging, inertial navigation and a video camera. However, the experiments are focused in outdoor navigation and are based in the data obtained by an Inertial Measure Unit (IMU) and a GPS device.

The IMU is a InertiaLink 3DM-GX2 unit [14] containing triaxial accelerometer, gyro and magnetometer. Only accelerometer and gyroscope readings will be used. The global position sensor is a Novatel® OEMV-1G differential GPS [15]. It is compatible with Satellite Based Augmentation Systems as EGNOS, though it has been operating on single point L1 mode for these experiments -accuracy of $1.5 \mathrm{~m}$ (RMS) in ground positioning.

The robotic platform is equipped with an embedded computer for highdemanding computing tasks. It also allows the integration of sensing and control hardware through the Player/Stage architecture [16]. 
External references are obtained only by means of a GPS -internal sensor-, so the platform will be subject to impoverished sampling frequencies and outage periods to mimic adverse conditions that can arise using external sensors.

\subsection{Fusion Performance}

This section presents the results of executing the proposed experiments with simulated and real trajectories. Special attention will be put in unexpected behaviors and other unusual effects, to help refining the system in future developments.

A stadium-shaped trajectory was used to calculate a general view of the overall filter performance. Receiving GPS measures at $1 \mathrm{~Hz}$, the PF can easily obtain an average $40 \%$ improvement over bare observations, as shown in Figure 4.

The last test use a circular trajectory to see how the system performs when only low-accuracy positioning is available in reduced spaces and the availability of external references is restricted. Figure 5. shows the obtained filtered trajectory. In

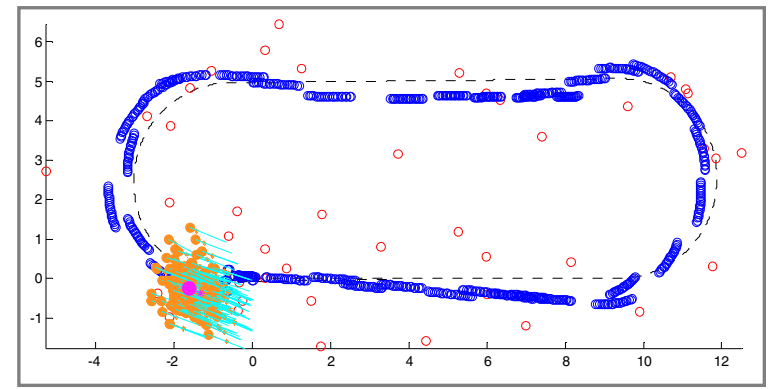

Fig. 4 In a stadium-shaped trajectory the PF improves GPS accuracy an average $40 \%$ (from meter-precision to $0.6 \mathrm{~m}$ ).

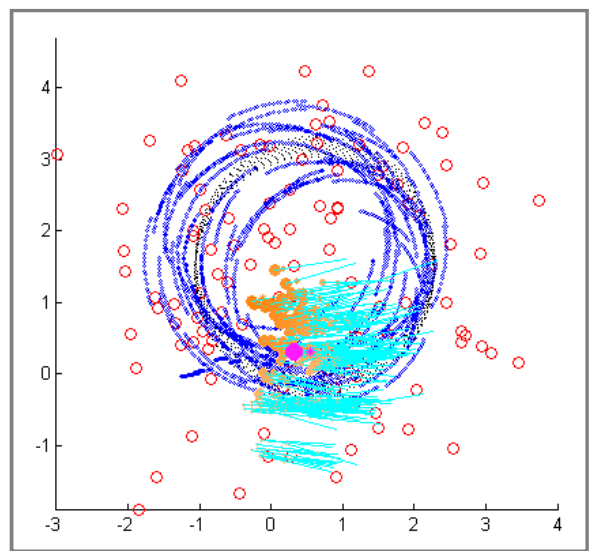

Fig. 5 Filter results using low accuracy positioning in reduced spaces

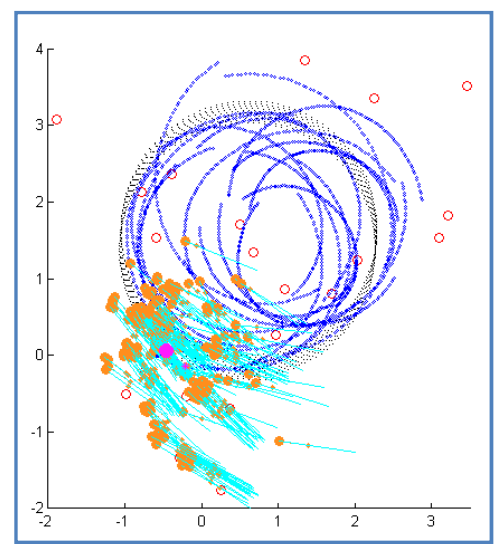

Fig. 6 Same experiment using a $0.1 \mathrm{~Hz}$ GPS signal 
spite of its positioning error below $0.55 \mathrm{~m}$-even better than the stadium case-,the motion pattern is erratic, with large orientation errors. Figure 6. Shows that the prediction is much worse when GPS measures arrive each 10 seconds. Particle population is largely scattered through state space, but the filter do not diverge.

\section{Conclusions and Future Work}

A system for robust navigation in both indoor and outdoor environments has been presented. Apart from being able to operate in standalone mode, it can interact with smart environments to take advantage of an extended sensory capability.

The architecture of the interaction system has been defined to support the simultaneous load of many robotic platforms, while being capable of dealing with any kind of failure. Moreover, mobile platforms using the proposed navigation system can integrate external sensors straightforwardly, without requiring any kind of configuration.

Smart environments including this system into their functionality only have to implement the multiagent system as an independent module. Its design is focused in not being intrusive and making a rationale use of resources, so that early functionality is not affected by the execution of the sensory services.

The platform has been subject to some preliminary tests, resulting in acceptable results even under conditions of reduced sensor availability. Future work include the integration of magnetometer, odometer and laser sensor as internal devices, and two external positioning systems: Ultra Wide Band and video tracking.

Some of these sensors have been included in other works [17] to achieve exceptional accuracy levels that we should be able to reproduce with reduced effort.

\section{Acknowledgment}

This work was supported in part by Projects CICYT TIN2008-06742-C02-02/TSI, CICYT TEC2008-06732-C02-02/TEC, CAM CONTEXTS (S2009/ TIC-1485) and DPS2008-07029-C02-02.

\section{References}

[1] Takahashi, Y., Kohda, M., Kanbayashi, Y., Yamahira, K., Hoshi, T.: Tray Carrying Robot for Hospital Use. IEEE, Los Alamitos (2005)

[2] Wulf, O., Lecking, D., Wagner, B.: Robust Self-Localization in Industrial Environments based on 3D Ceiling Structures. IEEE, Los Alamitos (2006)

[3] Cannon, M.E., Nayak, R., Lachapelle, G., Salychev, O.S., Voronov, V.V.: Low-Cost INS/GPS Integration: Concepts and Testing. The Journal of Navigation 54, 119-134 (2001)

[4] Gordon, N.J., Salmond, D.J., Smith, A.F.M.: Novel approach to nonlinear/non-Gaussian Bayesian state estimation. IEE Proceedings F Radar and Signal Processing 140, 107-113 (1993) 
[5] Huang, L., Barth, M.: Tightly-coupled LIDAR and computer vision integration for vehicle detection. IEEE, Los Alamitos (2009)

[6] Zampieron, J.: Self-Localization in Ubiquitous Computing using Sensor Fusion (2006)

[7] Gil, G., Berlanga de Jesús, A., Lopéz, J.M.: Multi-sensor and Multi Agents Architecture for Indoor Location. Distributed Computing and Artificial Intelligence - Advances in Soft Computing, 309-316 (2010)

[8] Rosencrantz, M., Gordon, G., Thrun, S.: Decentralized Sensor Fusion with Distributed Particle Filters. In: Proc. of UAI (2003)

[9] Welch, G., Bishop, G.: An Introduction to the Kalman Filter (1995)

[10] Arulampalam, M.S., Maskell, S., Gordon, N.: A tutorial on particle filters for online nonlinear/non-Gaussian Bayesian tracking, vol. 50, pp. 174-188 (2002)

[11] Gustafsson, F., Gunnarsson, F., Bergman, N., Forssell, U., Jansson, J., Karlsson, R., Nordlund, P.-J.: Particle filters for positioning, navigation, and tracking. IEEE Transactions on Signal Processing 50, 425-437 (2002)

[12] Merwe, R.V.D., de Freitas, N., Doucet, A., Wan, E.: The Unscented Particle Filter.

[13] Automation, R.: Guardian Mobile Robot datasheet (2010)

[14] MicroStrain, Inertia-Link ® 3DM-GX2 Datasheet (2008)

[15] Novatel, OEMV-1G GNSS Card Datasheet (2010)

[16] Gerkey, B.P., Vaughan, R.T., Howard, A.: The Player/Stage Project: Tools for MultiRobot and Distributed Sensor Systems, pp. 317-323 (2003)

[17] Cheng, Y., Crassidis, J.L.: Particle Filtering for Attitude Estimation Using a Minimal Local-Error Representation. Journal of Guidance, Control, and Dynamics 33, 1305-1310 